Mots. Les langages du politique

$85 \mid 2007$

Violence et démocratie en Amérique latine

\title{
Francine Mazière, L'analyse du discours. Histoire et pratiques
}

Marie-Anne Paveau

\section{CpenEdition \\ Journals}

Édition électronique

URL : https://journals.openedition.org/mots/1260

DOI : $10.4000 /$ mots. 1260

ISSN : 1960-6001

Éditeur

ENS Éditions

Édition imprimée

Date de publication : 1 novembre 2007

Pagination : 112-116

ISBN : 978-2-84788-113-4

ISSN : 0243-6450

Référence électronique

Marie-Anne Paveau, «Francine Mazière, L'analyse du discours. Histoire et pratiques », Mots. Les langages du politique [En ligne], 85 | 2007, mis en ligne le 01 novembre 2009, consulté le 22 avril 2022. URL

http://journals.openedition.org/mots/1260 ; DOI : https://doi.org/10.4000/mots.1260 
discours de Mendoza, el tiempo [del negativo] es un recurso que se agota [le temps [du négatif] est une ressource qui s'épuise] (p. 111), métaphore politiquement contre-productive car d'allure fataliste.

$\mathrm{Au}$ terme de ces lectures, on pourrait se demander si l'alternative est entre langue populiste et langue de bois. Quoi qu'il en soit, cette série d'articles bien ancrés dans les données et les méthodes linguistiques, cohérents et variés du point de vue des approches théoriques et, politiquement, globalement nuancés, propose une lecture à chaud, mais construite, du discours politique à une période clé de la vie sociale et institutionnelle du Venezuela. Cet ensemble constitue une ressource de référence pour l'étude du discours politique en général, et en particulier pour les questions d'émergence et de structuration du discours de confrontation, ainsi que pour l'étude des pratiques langagières de celui qui est, de facto, l'un des plus importants leaders d'opinion de l’Amérique Latine.

Christian Plantin

Université Lumière Lyon 2, ENS-LSH, CNRS (ICAR)

Christian.Plantin@univ-lyonz.fr

\section{L'analyse du discours. Histoire et pratiques}

Francine Mazière

2005, Paris, Presses universitaires de France (Que sais-je ?), 128 p.

Un «Que sais-je? » sur l'analyse du discours. Bigre. Voilà qui défie l'encyclopédisme de la légendaire petite collection, tant le domaine s'est apparemment étendu au-delà de ce qu'il semble raisonnable d'enserrer dans les 128 pages règlementaires. Et surtout, l'analyse du discours est depuis longtemps (toujours?) un domaine mis et remis en question, parfois sans ménagement, objet d'intenses projections imaginaires concernant la scientificité et l'identité disciplinaire : la linguistique de la langue lui reproche de prendre comme objet... le discours, et de quitter pour ainsi dire les bords nets de la linguistique tels qu'ils auraient été coupés par Saussure; la stylistique littéraire ne comprend pas bien pourquoi elle semble tenue à l'écart d'une discipline qui lui semble méthodologiquement bien ressemblante; l'interactionnisme lui reproche son monologisme et son gout pour les corpus écrits; les sémanticiens du texte critiquent son éclectisme méthodologique, sa faiblesse théorique et le choix superflu de cet objet «discours», toujours soupçonné d'usurper le véritable objet de la science du sens, le texte. On pourrait augmenter la liste en allant du côté de la psychologie sociale, de la sémiotique, etc. Autant dire que le terrain sur lequel s'est aventurée Francine Mazière est quelque peu miné, et que tout discours sur l'analyse du discours est de toute façon et structurellement susceptible de 
recevoir quelques critiques bien senties. Comme c'est le propre de cette collection de permettre toutes sortes de synthèses, pourvu qu'elles soient cohérentes, clairement exposées et qu'elles apportent honnêtement les connaissances nécessaires à tout un chacun sur la question qui l'intéresse, l'auteure a fait un certain nombre de choix pour répondre à la célèbre question posée par la collection. Sans éviter tout à fait certaines ornières, elle livre une mise au point qui nous semble convaincante et surtout nécessaire.

Francine Mazière circonscrit très précisément sa perspective en signalant que son «objectivité est [...] celle d'un point de vue et d'un témoignage» (p. 4). On comprend donc que l'auteure est également actrice de l'histoire qu'elle se propose de retracer, en particulier autour d'évènements scientifiques précis, entre 1966 et 1983, période bornée par les premières publications et la mort de Michel Pêcheux. Elle précise bien le champ d'investigation de l'ouvrage qui «s'appuie essentiellement sur la littérature développée au sein des sciences du langage, la linguistique demeurant le principal lieu institutionnel de l'AD» (p. 3). Elle met d'ailleurs l'ouvrage sous les auspices de Zellig S. Harris, dont elle cite d'emblée le fameux texte de 1952 (voir références infra). Cela implique que les approches (psycho)sociologiques, communicationnelles, ethnométhodologiques ou argumentatives ne seront pas centrales dans son approche. Bien sûr, on entend déjà les reproches à l'encontre de cette posture : subjectivité et restriction de champ. Mais il y a belle lurette que les travaux de sociologie des sciences et d'épistémologie ont montré que l'objectivité universalisante n'était jamais qu'une intersubjectivité consensuelle; ensuite, il n'existe pas encore, jusqu'à présent, d'histoire de l'analyse du discours dite «française $»^{1}$, c'est-à-dire autour du groupe de travail qu'anima Michel Pêcheux, à partir d'une réflexion linguistique inventive dont les travaux en cours ${ }^{2}$ montreront la force théorique et la fécondité parfois souterraine dans différentes traditions qui se réclament actuellement de l'analyse du discours. Le «Que saisje? » de Francine Mazière accomplit donc fort bien sa mission encyclopédique, en rendant compte d'un segment de vingt ans d'histoire intellectuelle et théorique française qui est sur le point de tomber dans l'oubli.

L'auteure rappelle (chapitre 1) l'importance des concepts fondateurs de l'analyse du discours, en particulier le corpus («construction d'un dispositif d'observation», p.11, et non simple relevé ou liste d'énoncés) et la langue,

1. On dispose pour l'instant des écrits que Denise Maldidier a consacrés à Michel Pêcheux (1990; 1993); des travaux de Jacques Guilhaumou sur certains concepts de l'analyse de discours comme la formation discursive (2005, www.marges-linguistiques.com, p. 95-114, consulté le 26 juillet 2005) ; des deux dictionnaires parus en 2001 (Détrie, Siblot, Vérine) et 2002 (Charaudeau, Maingueneau), qui donnent cependant très peu d'indications historiques; de deux articles de JeanJacques Courtine (1991, p. 153-171; 2003); enfin d'un article récent de Christian Puech (2005, p. 93-110) qui propose malheureusement une vision microscopique de la théorie du discours de Pêcheux confrontée aux conceptions de Foucault.

2. Paveau, Rosier, 2005 ; Rosier, 2005 ; Paveau, à paraitre. 
abordée à la fois comme structure et comme production empirique des acteurs sociaux, ce qui est sans doute une spécificité de la discipline - on y reviendra. Elle signale également que le concept de sujet, au cœur de l'approche marxiste de Pêcheux, a été pensé et repensé, dans la diversité et la discussion, par les différentes approches qui ont succédé à la première configuration théorique (sujet dispersé de Foucault, sujet de l'inconscient pour la tendance psychanalytique, sujet de l'histoire, sujet de l'énonciation, sujet de l'acte de langage pour la pragmatique). La question des savoirs de l'analyste est également examinée, directement liée à celles du corpus et du sujet, ainsi que celle des types de discours et du traitement du sens. Corpus, langue, sujet, savoirs, types et sens : voilà un petit vade mecum certes restrictif, mais assez dense et opératoire pour comprendre les principes théoriques et méthodologiques de l'analyse du discours sans dispersion. Sur ce point, le pari encyclopédique nous semble gagné.

Les développements historiques, balisés d'abord par les travaux de Jean Dubois $^{3}$ (chapitre 2), proposent une lecture détaillée de l'apparition du syntagme analyse du discours en France en 1969 (Langages, $n^{\circ}{ }_{13}$ ) ainsi que des méthodes et des soubassements théoriques impliqués par un véritable travail d'importation scientifique : passage des classes d'équivalence harrissiennes aux classes propositionnelles (ce qui implique le choix méthodologique du mot-pivot), évolution de l'analyse formelle (distribution et transformation) vers l'interprétation sémantique qui prend en compte le sujet comme lieu du texte (Dubois, 1969, p. 100-110), interrogations sur l'articulation des structures de surface et des structures profondes dans un cadre grammairien qui ne met jamais la langue en doute.

La description des travaux autour d'une autre figure fondatrice de l'analyse du discours dite «française», Michel Pêcheux (chapitre 3), que l'auteure qualifie avec justesse de "lieu générique» (p. 45), montre par quels chemins est passée la théorie du discours : à partir d'un horizon philosophique (le premier ouvrage de Pêcheux porte sur la philosophie des sciences), le discours est travaillé dans le cadre du marxisme althussérien et de la psychanalyse lacanienne, dans une perspective linguistique forte - celle de la linguistique culiolienne qui permet des hypothèses sur la formalisation en linguistique. Complexité de l'objet ou manque de clarté dans l'analyse, ce passage sur Pêcheux est malaisé à suivre, les inventions et les expérimentations du groupe se superposant parfois : on aurait préféré un cheminement plus chronologique, retraçant les évolutions de Pêcheux, ou alors thématique, prenant les concepts les uns après les autres (intradiscours, interdiscours, préconstruit, formation discursive, etc.). En l'état, le chapitre fourmille de références, de prolongements, d'analogies, et semble hésiter entre la monographie et la description collective d'un courant de

3. Dubois, 1968; avec Sumpf éd., 1969; 1971. 
recherches. Il fallait assurément faire les deux, mais peut-être successivement. Cependant, les acquis de cette période lue par Francine Mazière méritent d'être relevés, en particulier parce qu'ils se démarquent, par leur richesse conceptuelle, d'une vision plus ordinaire de l'analyse du discours : transdisciplinarité, langue, matérialité discursive, hétérogénéité, réseau de mémoire, dictionnaire discursif. Mais aussi parce que, comme le dit très bien l'auteure, «l'AD est un bon exemple du glissement des concepts sans généalogie, alors même qu'elle ne s'est conçue que par et dans son histoire» (p. 63).

Le dernier chapitre de l'ouvrage porte sur la pluridisciplinarité centrée autour de la sociolinguistique et de l'histoire, ainsi que sur les pratiques actuelles en analyse du discours. Francine Mazière rappelle d'abord les enjeux d'un débat important entre sociolinguistique et analyse du discours (fin des années soixantedix), la question de la langue étant, encore et toujours, au cœur des interrogations et des affrontements. Les développements de la discipline via les apports de la sociologie du langage, de la sociolinguistique variationniste, de l'ethnométhodologie alimentent, dans les années quatre-vingt, une conception sociale de la langue qui remet en cause l'axiome saussurien. On comprend donc que l'évolution de l'analyse du discours implique des modifications épistémologiques fortes, au-delà des choix de corpus et de méthodes. Francine Mazière rappelle également que les interrogations sur l'interdisciplinarité des années quatrevingt-dix (ethnologie et linguistique, sociologie et linguistique) ont souvent été vives, voire polémiques : articulation malaisée entre les méthodes et les conceptions (la conception de la langue de l'ethnologue et du sociologue ne s'accordant pas forcément avec celle du linguiste), accumulation de courants et de croisements disciplinaires n'évitant pas le danger de la dilution théorique. Au milieu de tous ces débats et des travaux de plus en plus nombreux qui se développent, la question de la langue et de la linguistique est continuellement retravaillée : «[...] les chercheurs prennent acte d'une certaine déchirure de la linguistique et de la fin des respects qu'on pensait lui devoir» (p. 83) ; déchirure de la linguistique qui est aussi celle de la langue saussurienne, bien à l'étroit désormais dans les pratiques contemporaines. Francine Mazière pense à juste titre que, si l'analyse du discours n'a pas forcément réussi à décrire ce que devient la langue dans le discours - ou, point de vue différent mais qui pose la même question, ce que le discours fait à la langue -, «l'AD a sans doute imposé une consistance, celle de la matérialité langagière, constitutive du sens, celle d'un sujet dans et par la langue» (p. 85).

Le chapitre se clôt sur une description des activités des chercheurs en analyse du discours dans les structures de recherche française. On se risquera à dire que Francine Mazière, en bonne historienne de la linguistique, est plus à l'aise dans le récit du passé que l'analyse du présent. Elle ne cite en effet que trois centres de recherche, à Paris et en région parisienne (Céditec de Paris 12, Cediscor de Paris 3, CAD de Paris 13), pour de bonnes raisons, certes, puisque 
le format du «Que sais-je?» ne lui permet guère les développements. Mais l'analyse du discours est active depuis bien longtemps à Montpellier 3 par exemple (l'UMR Praxiling autour de la praxématique et l'équipe Dipralang autour des approches sociolinguistiques), et il s'agit d'un centre important d'élaboration théorique, de publication et de formation de jeunes chercheurs. On pense également au GRIC de Lyon 2 sur les interactions (structure absorbée désormais dans l'UMR ICAR) ou des travaux autour de R. Vion à Aix-en-Provence. Une petite erreur sur la nature des travaux (et le sexe...) de Dominique Ducard confirme ces hésitations. Enfin les annexes, dues à André Collinot, ne nous semblent guère représentatives des "écritures d'analyse du discours", expression qui constitue cependant un bien beau programme. Certains travaux retenus sont en effet trop confidentiels et trop « micro » pourillustrer les éléments précédemment évoqués dans le corps du livre. Il eût fallu à notre avis choisir uniquement des ouvrages aisément consultables par les lecteurs français et étrangers et représentatifs de la discipline. Cela d'autant plus que l'auteure ne fournit pas de bibliographie à son «Que sais-je? » pour des raisons de place, ce qui constitue selon nous un manque : il était possible de donner une trentaine de titres, ne serait-ce qu'en reprenant les travaux «princeps » mentionnés dans le corps du texte. En bref, la fin de l'ouvrage laisse le lecteur un peu perplexe et sur sa faim.

Il reste que ce petit livre, tel qu'il est pensé et écrit, orienté et lesté des témoignages et de l'expérience de l'auteure, a sa place dans la description, encore rare, d'un des domaines disciplinaires les plus riches de l'histoire intellectuelle française.

Marie-Anne Paveau

Université Paris 13, Céditec Paris 12

marie-anne.paveau@libertysurf.fr

\section{Références}

Charaudeau Patrick, Maingueneau Dominique, 2002, Dictionnaire d'analyse du discours, Paris, Le Seuil.

Courtine Jean-Jacques, 1991, «Le discours introuvable : marxisme et linguistique (19651985) ", Histoire épistémologie langage, $\mathrm{n}^{\circ} 13$ (2), Théories et données, p. 153-171.

— 2003, "L'étrange mémoire de l'analyse du discours», texte en français à paraitre en portugais dans les Actes du colloque de Porto Alegre pour les vingt ans de la disparition de Michel Pêcheux (trad. Carlos Piovezani).

DÉTRIE Catherine, SIB LOT Paul, VÉRINE Bertrand, 2001, Termes et concepts pourl'analyse du discours, Paris, Champion.

Duвoıs Jean, 1968, «Lexicologie et analyse d'énoncés», Cahiers de lexicologie, $\mathrm{n}^{\circ} 15$, p. 115-126.

— 1969, «Énoncé et énonciation », Langages, n 13, p. 100-110. 
— 1971, "L'analyse de discours», préface à la thèse de Jean-Baptiste Marcellesi, Le Congrès de Tours (décembre 1920). Études sociolinguistiques, Paris, Le Pavillon, p. 15-19.

Dubois Jean, SumpF Joseph éd., 1969, Langages, $\mathrm{n}^{\circ}$ 13, Analyse de discours.

Guilhaumou Jacques, 2005, "Où va l'analyse de discours? Autour de la notion de formation discursive », Marges linguistiques, $\mathrm{n}^{\circ}$ 9, Analyse du discours. État de l'art et perspectives, www.marges-linguistiques.com, p. 95-114, consulté le 26 juillet 2005.

HARRIS Zellig S., 1952, «Discourse analysis : a sample text», Language, $n^{\circ} 28$ (4), traduit dans J. Dubois, J. Sumpf éd., Langages, $\mathrm{n}^{\circ}$ 13, p. 8-45.

Maldidier Denise, 1990, L'inquiétude du discours. Textes de Michel Pêcheux, Paris, Éditions des Cendres.

- 1993, "L'inquiétude du discours. Un trajet dans l'histoire de l'analyse du discours : le travail de Michel Pêcheux», Semen, $n^{\circ} 8$, Configurations discursives.

PAVEAu Marie-Anne, Rosier Laurence, 2005, «Éléments pour une histoire de l'analyse du discours. Théories en conflit et ciment phraséologique», communication au colloque franco-allemand: "L'analyse du discours en France et en Allemagne. Tendances actuelles en sciences du langage et sciences sociales», Créteil, Céditec, 2 juillet, texte consultable sur http://www.johannes-angermueller.de/francais/adfa.html

PAVEAu Marie-Anne, à paraitre, "Analyse du discours et histoire. Rencontres et oublis»,

S. Bonnafous, M. Temmar éd., Analyse du discours et sciences humaines, Paris, Ophrys.

PueCH Christian, 2005, «L'émergence de la notion de discours en France et les destins du saussurisme », Langages, $\mathrm{n}^{\circ} 159$, p. 93-110.

ROSIER Laurence, 2005, «Analyse du discours et sociocritique », Littérature, p. 34-47.

\section{La presse illustrée au xix ${ }^{\mathrm{e}}$ siècle. Une histoire oubliée}

Jean-Pierre Bacot

2005, Limoges, Presses universitaires de Limoges

L'étude, par Jean-Pierre Bacot, de la presse illustrée qui nait au $19^{\mathrm{e}}$ siècle en Europe lui permet de la concevoir comme un objet propre et autonome. Elle se démarque en effet par sa temporalité, distincte de celle de la presse classique comme de celle de l'histoire générale, et par ses conditions d'émergence et de diffusion. Les développements techniques (des transports avec le chemin de fer comme de l'édition avec la technique de gravure "bois debout»), et surtout les mutations sociales et politiques des sociétés européennes, ont permis l'avènement d'une presse illustrée en Europe, mais ce sont les transformations spécifiques de la société anglaise qui en composeront le terreau nécessaire. Tandis que la France se singularise par le dynamisme de la presse non illustrée avec la naissance de la première agence de presse, fondée par Charles Havas en 1835, l'Angleterre sera le lieu de la modélisation de ce que l'auteur définit comme les premières générations de presse illustrée, qui se diffuseront ensuite largement sur le 